\section{Effect of Chronic Sham Feeding on Maximal Gastric Acid Secretion in the Dog}

Richard C. Thirlby and Mark Feldman

University of Texas Health Science Center at Dallas and Dallas Veterans Administration Medical Center,

Dallas, Texas 75216
A acid secretory capacity in some patients with duodenal ulcer is uncertain. We postulated that chronically increased cephalic-vagal stimulation may be a cause of increased maximal acid output. To study this, we prepared six male, mongrel dogs with a vagally innervated gastric fistula, a vagally denervated fundic (Heidenhain) pouch, and a cervical esophagostomy. Physiological cephalic-vagal stimulation was accomplished by sham feeding, which increased acid output from the vagally innervated stomach but not from the vagally denervated pouch. During an initial 6-wk control period, dogs were fed by mouth once daily at 3 p.m. Then, a 6 -wk period of sham feeding was carried out, during which animals were sham fed with blenderized dog chow from 8 a.m. to 3 p.m. every day (a 7-h period of continuous cephalic-vagal stimulation), after which animals were fed by mouth. After 6 wk of daily sham feeding, maximal acid output in response to intravenous pentagastrin $(16 \mu \mathrm{g} / \mathrm{kg}$ per $\mathrm{h})$ increased by $27 \pm 4 \%$ in the vagally innervated stomach $(P<0.01)$. Maximal acid output then returned to control levels after a final 6-wk recovery period with no sham feeding. No changes in maximal acid output occurred in the vagally denervated pouch during the 18-wk study. No changes in basal acid secretion or responsiveness of parietal cells to submaximal doses of pentagastrin occurred in the fistula or pouch during chronic sham feeding. We conclude that chronic physiological cephalic-vagal stimulation can increase maximal acid secretory capacity. Our studies also

Part of this work was presented at the Plenary Session of the American Gastroenterological Association in Washington, DC on May 24, 1983 and was published in abstract form in 1983, Gastroenterology, 84:1334.

Received for publication 25 July 1983 and in revised form 14 September 1983.

\section{J. Clin. Invest.}

(c) The American Society for Clinical Investigation, Inc. 0021-9738/84/02/0566/04

Volume 73, February 1984, 566-569 suggest that the effect of chronically increased vagal stimulation on maximal acid secretory capacity is reversible.

\section{Introduction}

Patients with duodenal ulcer, as a group, have an increased basal acid output and maximal acid output (MAO) $)^{1}(1-5)$. There is evidence that increased basal acid output in some patients with duodenal ulcer is due to increased cephalic-vagal stimulation (increased basal vagal tone) (1-4). The cause of increased MAO in duodenal ulcer patients is unknown.

We postulated that chronically increased cephalic-vagal stimulation might also be responsible for an increased MAO in some patients with duodenal ulcer. In order to study this hypothesis, we developed an animal model for chronic cephalicvagal stimulation using chronic sham feeding. Our purpose was to determine whether chronic cephalic-vagal stimulation in the dog would lead to an increased pentagastrin-stimulated MAO.

\section{Methods}

Animal preparation. Six male mongrel dogs (21-28 kg) were prepared with a vagally innervated gastric fistula drained by a Thomas cannula, a vagally denervated fundic (Heidenhain) pouch drained by a Gregory cannula, and an exteriorized cervical esophagostomy (6). The esophagostomy was fitted with a specially designed plastic cannula that could be occluded with a plug to allow normal feeding. Dogs were housed in individual kennels and acid secretory studies were started no earlier than $3 \mathbf{w k}$ after recovery from surgery.

Acute sham feeding experiments. After an 18-h fast, dogs were placed in a Pavlov sling. Basal secretions were collected by gravity for four 15min periods. Then, the cervical esophagostomy was unplugged and the cervical esophagus just distal to the esophagostomy was occluded with a nylon cord tourniquet. Sham feeding was carried out for $10 \mathrm{~min}$ using blenderized dog chow (Wayne Lab dog diet, Allied Mills, Fort Wayne, IN). Secretions from the gastric fistula and fundic pouch were collected for four additional 15 -min periods. Then, $6 \mu \mathrm{g} / \mathrm{kg}$ pentagastrin (Peptavlon, Ayerst Labs, New York) was injected subcutaneously and acid secretion measured for four additional 15 -min periods.

The volume of each 15-min sample of gastric juice was measured and hydrogen ion concentration $\left[\mathrm{H}^{+}\right]$determined by glass electrode (7). Acid output was calculated by multiplying volume by acid concentration.

1. Abbreviations used in this paper: MAO, maximal acid output. 


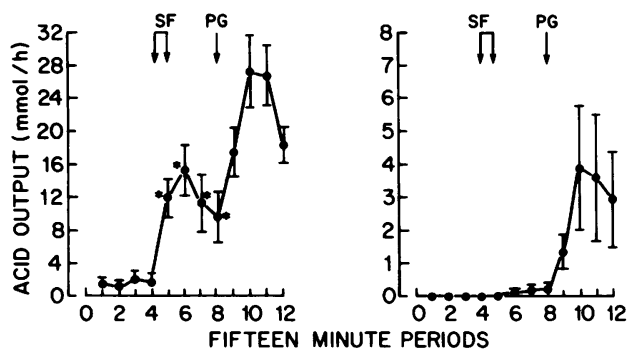

Figure 1. Effect of acute sham feeding (SF) and $6 \mu \mathrm{g} / \mathrm{kg}$ subcutaneous pentagastrin (PG) on mean $( \pm \mathrm{SE})$ acid outputs from vagally innervated gastric fistula (left) and vagally denervated fundic pouch (right) in six dogs. Sham feeding increased acid output significantly $(*, P$ $<0.05$ ) in the vagally innervated gastric fistula but not in the vagally denervated fundic pouch. The denervated fundic pouch did respond, however, to the near-maximal dose of pentagastrin.

As shown in Fig. 1 (left), sham feeding led to a large increase in mean acid output from the vagally innervated gastric fistula, amounting to $54 \%$ of the response to $6 \mu \mathrm{g} / \mathrm{kg}$ pentagastrin. On the other hand, acid output from the vagally denervated pouch did not increase significantly in response to sham feeding and remained $<5 \%$ of the response to 6 $\mu \mathrm{g} / \mathrm{kg}$ pentagastrin (Fig. 1 , right). This confirms previous findings that sham feeding stimulates gastric acid secretion via vagal pathways to the stomach (8).

Chronic sham feeding protocol. An 18-wk protocol was used to evaluate the effect of chronic cephalic-vagal stimulation on MAO and also on the responsiveness of parietal cells to submaximal doses of pentagastrin. Normal feeding was carried out once per day at $3 \mathrm{p}$.m. throughout the 18-wk protocol. The first 6 wk was a control period with normal feeding but no sham feeding. The second 6 wk was a chronic sham feeding period. Dogs were sham fed every day with blenderized chow from 8 a.m. to 3 p.m., a 7-h period of continuous cephalic-vagal stimulation, followed by normal feeding at $3 \mathrm{p}$.m. Continuous sham feeding was made possible by filling a $24 \times 24-"$ pan with blenderized chow and by refilling the pan as needed. Swallowed food exited from the esophagostomy into the pan and thus was available for reingestion and continuous sham feeding. The dogs did not lose interest in the food and sham fed during the 7-h period with only occasional pauses. After 6 wk of chronic sham feeding, dogs entered a third 6-wk recovery period with normal feeding at 3 p.m. and no sham feeding.

Pentagastrin-stimulated acid secretion was determined six times during the 18-wk protocol: at 0 and 6 wk of the control period; at 8,10 , and 12 wk during the chronic sham feeding period; and at $18 \mathrm{wk}$, at the end of the recovery period. Studies were performed after a $24-\mathrm{h}$ fast and without simultaneous sham feeding. During these acid secretory studies, dogs stood on tables and were supported by Pavlov slings. Basal secretions were collected for three 10-min periods from the gastric fistula and fundic pouch. Then, pentagastrin dissolved in $154 \mathrm{mM} \mathrm{NaCl}$ was infused by a pump through a polyethylene catheter in a peripheral leg vein (volumetric infusion pump model 922; IMED Corp., San Diego, CA). Pentagastrin was infused in a step-dose fashion, each dose $(1,2$, 4,8 , and $16 \mu \mathrm{g} / \mathrm{kg}$ per h) infused for three 10 -min periods. Acid output for each dose was the sum of the three consecutive 10-min outputs (multiplied by two to express results in millimoles per hour). In dogs, either 4,8 , or $16 \mu \mathrm{g} / \mathrm{kg}$ per $\mathrm{h}$ pentagastrin have been reported as the maximally effective dose of pentagastrin $(9,10)$. Because in our present studies $16 \mu \mathrm{g} / \mathrm{kg} / \mathrm{hr}$ pentagastrin produced acid outputs that were sig- nificantly higher than outputs after 4 or $8 \mu \mathrm{g} / \mathrm{kg}$ per h $(P<0.05)$, only acid outputs for the $16 \mu \mathrm{g} / \mathrm{kg} / \mathrm{hr}$ pentagastrin dose will be referred to as MAO in this report.

In each of the six dogs, fasting venous blood was collected for gastrin measurement four times: at $6 \mathrm{wk}$ of the control period; at 9 and $12 \mathrm{wk}$ during the chronic sham feeding period; and at $18 \mathrm{wk}$, at the end of the recovery period. Serum gastrin concentrations were kindly measured by radioimmunoassay by Walsh (11) using previously described methods.

Statistics. Data are expressed as mean \pm one standard error. Differences in mean data were tested for statistical significance by paired $t$ test. $P$ values less than 0.05 were considered statistically significant.

\section{Results}

\section{Acid secretion}

$M A O$. Mean $( \pm \mathrm{SE})$ MAO from the vagally innervated gastric fistula in response to $16 \mu \mathrm{g} / \mathrm{kg}$ per h pentagastrin in six dogs is shown in Fig. 2. MAO averaged $25.7 \pm 2.4$ and $27.5 \pm 2.3 \mathrm{mmol} /$ $h$ at the beginning and end of the 6-wk control period, respectively $(P>0.4)$. MAO increased progressively and significantly during the chronic sham feeding period. MAO averaged 33.5 \pm 2.4 $\mathrm{mmol} / \mathrm{h}$ at $12 \mathrm{wk}$ (i.e., after $6 \mathrm{wk}$ of chronic sham feeding), increasing above both of the control MAO values in every dog $(P<0.05)$. MAO increased from 10 to $35 \%$ in the six dogs (mean \pm SE, $27 \pm 4 \%$ ). At the end of the 6-wk recovery period (i.e., at $18 \mathrm{wk}$ ), MAO had decreased below the MAO at $10 \mathrm{wk}$ and at $12 \mathrm{wk}$ in each $\operatorname{dog}(P<0.05)$, with a mean MAO of $26.7 \pm 2.2 \mathrm{mmol} / \mathrm{h}$ (Fig. 2).

There were no significant changes in MAO from the vagally denervated fundic pouch during the 18-wk study (Fig. 2).

Responsiveness to submaximal doses of pentagastrin. Mean acid outputs in response to 0 (basal), 1, 2, 4, and $8 \mu \mathrm{g} / \mathrm{kg}$ per

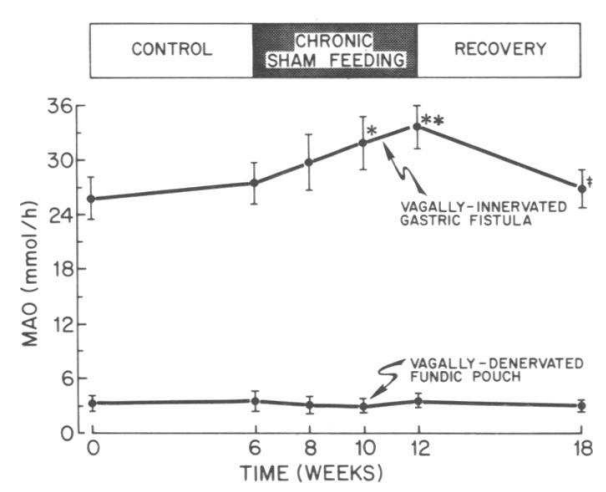

Figure 2. Mean $( \pm \mathrm{SE}) \mathrm{MAO}$ to $16 \mu \mathrm{g} / \mathrm{kg}$ per h pentagastrin i.v. in six dogs during 18-wk study protocol from the vagally innervated gastric fistula and vagally denervated fundic pouch. During the chronic sham feeding period, mean MAO increased significantly in the vagally innervated gastric fistula but not in the vagally denervated fundic pouch $(*, P<0.05$ vs. both control MAO values by one-tailed $t$ test, **, $P<0.01$ vs. both control MAO values by two-tailed $t$ test). By the end of the recovery period (i.e., at $18 \mathrm{wk}$ ), MAO decreased significantly ( $\ddagger, P<0.05$ vs. MAO values at 10 and 12 wk by twotailed paired $t$ test) and had returned to near control MAO values. 
h pentagastrin, expressed as a percentage of MAO to $16 \mu \mathrm{g} /$ $\mathrm{kg}$ per $\mathrm{h}$ pentagastrin, are shown in Fig. 3 for the vagally innervated gastric fistula. The upper left panel illustrates pentagastrin dose-response curves at the beginning and at the end of the 6-wk control period. These two curves were similar, both in the vagally innervated stomach (Fig. 3) and in the vagally denervated stomach (data not shown). Thus, responsiveness to submaximal doses of parenteral pentagastrin in the vagally innervated and the vagally denervated stomach was constant during the 6-wk control period.

During the chronic sham feeding period, there was no significant change in basal acid secretion or in responsiveness to submaximal doses of pentagastrin in the vagally innervated gastric fistula (Fig. 3) or vagally denervated pouch (data not shown). As shown by the asterisks, the pentagastrin dose-response curve for the vagally innervated gastric fistula was shifted significantly to the left of control at $18 \mathrm{wk}$ (Recovery, Fig. 3). There was no significant shift in the pentagastrin dose-response curve for the vagally denervated fundic pouch at this time.

\section{Serum gastrin concentration}

Fasting serum gastrin concentrations were unchanged throughout the 18-wk protocol. Mean $( \pm \mathrm{SE})$ values were $91 \pm 10 \mathrm{pg} / \mathrm{ml}$ at $6 \mathrm{wk}$ (control period), $85 \pm 9$ and $87 \pm 12 \mathrm{pg} / \mathrm{ml}$ at 9 and 12 wk (chronic sham feeding period), and $93 \pm 9 \mathrm{pg} / \mathrm{ml}$ at $18 \mathrm{wk}$ (end of recovery period).

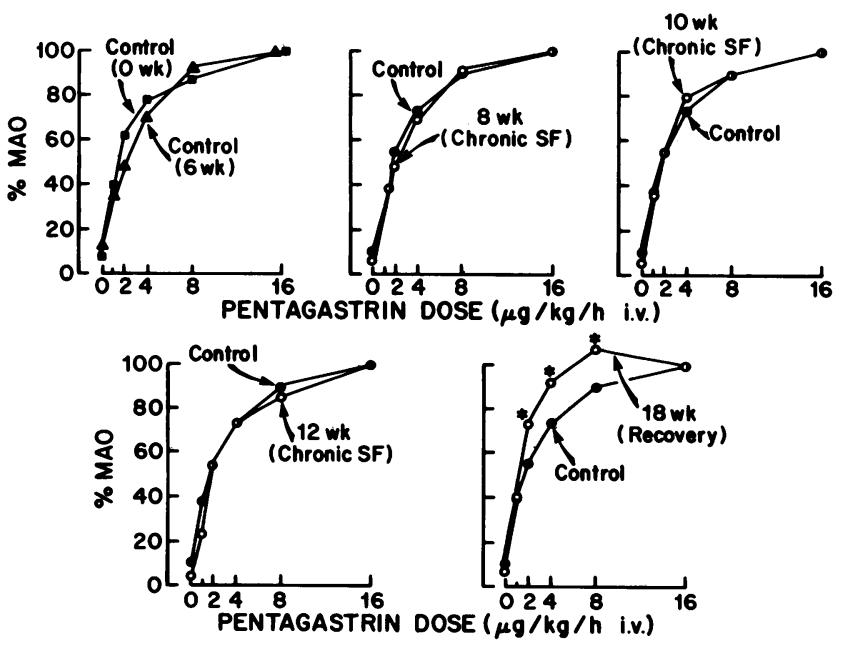

Figure 3. Mean intravenous pentagastrin-stimulated acid output, expressed as a percentage of MAO to $16 \mu \mathrm{g} / \mathrm{kg}$ per h pentagastrin, from the vagally innervated gastric fistula in six dogs. Pentagastrin dose-response curves at 0 and $6 \mathrm{wk}$ of the control period were similar and were combined in the other panels for comparison with dose-response curves at 8,10 , and 12 wk during chronic sham feeding (SF) and at $18 \mathrm{wk}$, at the end of the recovery period. No significant changes occurred during chronic SF, although the pentagastrin doseresponse curve was shifted to the left significantly at $18 \mathrm{wk}$ (end of the recovery period, $*, P<0.05$ ).

\section{Discussion}

The major finding in this study was that $7 \mathrm{~h}$ of continuous sham feeding per day for $6 \mathrm{wk}$ led to a $27 \%$ increase in MAO to pentagastrin. The increase in MAO, which occurred in every dog, was seen only in the vagally innervated gastric fistula and not in the vagally denervated fundic (Heidenhain) pouch (Fig. 2). These observations suggest that the increase in MAO during chronic sham feeding was mediated by direct vagal stimulation of parietal cells. If chronic sham feeding increased MAO by releasing a trophic hormone, such as gastrin (12), MAO would have been expected to increase also in the vagally denervated pouch, and it did not. Despite an increase in MAO in the vagally innervated stomach during chronic sham feeding, there was no change in basal acid secretion or in the responsiveness of parietal cells to submaximal doses of pentagastrin when acid secretion was expressed as a percentage of MAO. The $D_{s 0}$ to pentagastrin remained $\sim 1.5-2.0 \mu \mathrm{g} / \mathrm{kg}$ per $\mathrm{h}$ throughout the control and chronic sham feeding period.

MAO is believed to reflect parietal cell mass (13), and patients with increased MAO have parietal cell hyperplasia $(14,15)$. Thus, an increased MAO in response to chronic sham feeding in the vagally innervated stomach in this study may indicate that chronically increased cephalic-vagal stimulation led to parietal cell hyperplasia. In support of this concept is a study in cats by Pearl et al. (16) in which electrical stimulation of the anterior hypothalamus for $20 \mathrm{~h} / \mathrm{d}$ increased, within a few days, the density of gastric parietal, chief, and mucous neck cells. The gastric trophic response to anterior hypothalamic stimulation did not occur in vagotomized cats and therefore was probably vagally mediated. Although gastric acid secretion was not measured, peptic ulcers sometimes developed if electrical hypothalamic stimulation was continued for 4-6 wk (16).

At the end of a 6-wk recovery period, MAO decreased in every dog to near control values (Fig. 2). The mechanism for this decrease in MAO after cessation of chronic cephalic-vagal stimulation is unclear, but similar changes in MAO have been reported in patients with peptic ulcer who have been removed from a highly stressful environment (17). A decrease in MAO can also be produced by vagotomy, but not by chronic anticholinergic therapy $(18,19)$, suggesting that vagal mechanisms regulating MAO may not necessarily be cholinergic. Failure to restore MAO after proximal gastric vagotomy to prevagotomy levels with cholinergic agonists supports this suggestion $(20,21)$.

It is of interest that, at the end of the 6-wk recovery period, the pentagastrin dose-response curve in the vagally innervated fistula (but not in the denervated pouch) shifted to the left significantly (Fig. 3). For example, $2 \mu \mathrm{g} / \mathrm{kg}$ per h pentagastrin resulted in $55 \pm 5 \%$ of MAO during the control period and $74 \pm 3 \%$ of MAO at the end of the recovery period $(P<0.05)$. With 4 $\mu \mathrm{g} / \mathrm{kg}$ per h pentagastrin, these values were $74 \pm 3 \%$ vs. $92 \pm 3 \%$ of MAO, respectively $(P<0.02)$. In a study of three rhesus monkeys with vagally innervated gastric fistulae, Hall and Smith (22) found a similar leftward shift in the histamine dose-response 
curve during chronic (9-27 d) anterior hypothalamic stimulation. The leftward shift persisted poststimulation, but eventually was reversible (22). Why a leftward shift in the pentagastrin doseresponse curve in our study occurred after, but not during, chronic sham feeding is not certain.

The clinical implication from these studies is that chronically increased cephalic-vagal stimulation may be capable of increasing gastric acid secretion by increasing maximal secretory capacity. It is possible that chronically increased endogenous cephalicvagal stimulation, perhaps initiated by emotional stress, contributes to increased maximal acid secretion and parietal cell mass in some patients with peptic ulcer disease. The experimental findings in the dog also suggest that the effects of chronically increased vagal stimulation on maximal gastric acid secretion are reversible if the vagal stimulus is removed.

\section{Acknowledgments}

The authors wish to thank June Ferrarri, Dale Prince, Willie McFarland, George Lantrip, Steve Mitchell, and Kathy Smith for technical assistance and Vicky Slagle for preparing the manuscript.

This work was supported in part by grants AM 16816 and AM 26794 from the National Institute of Arthritis, Metabolism, and Digestive Diseases and by the Research Service of the Veterans Administration.

\section{References}

1. Dragstedt, L. R. 1969. Role of the vagus nerves in the physiology of gastric secretion. Am. J. Surg. 117:813-821.

2. Kirkpatrick, P. M., and B. I. Hirschowitz. 1980. Duodenal ulcer with unexplained marked basal gastric acid hypersection. Gastroenterology. 79:4-10.

3. Schwartz, T. W., B. Steinquist, L. Olbe, and F. Stadil. 1979. Synchronous oscillations in the basal secretion of pancreatic-polypeptide and gastric acid. Gastroenterology. 76:14-19.

4. Feldman, M., C. T. Richardson, and J. S. Fordtran. 1980. Effect of sham feeding on gastric acid secretion in healthy subjects and duodenal ulcer patients. Evidence for increased basal vagal tone in some ulcer patients. Gastroenterology. 79:796-800.

5. Grossman, M. I., J. B. Kirsner, and I. E. Gillespie. 1963. Basal and histalog-stimulated gastric secretion in control subjects and in patients with peptic ulcer or gastric cancer. Gastroenterology. 45:14-26.

6. Olbe, L. 1959. Esophageal cannula dog, a simple mode of preparation for sham feeding experiments. Gastroenterology. 32:460-462.
7. Moore, E. W., and R. W. Scarlata. 1965. The determination of gastric acidity by the glass electrode. Gastroenterology. 49:178-188.

8. Farrell, J. I. 1928. Contributions to the physiology of gastric secretion. The vagi as the sole efferent pathway for the cephalic phase of gastric secretion. Am. J. Physiol. 85:685-687.

9. Emas, S., A. Billings, and M. I. Grossman. 1968. Effects of gastrin and pentagastrin on gastric and pancreatic secretion in dogs. Scand. $J$. Gastroenterol. 3:234-240.

10. Keuppens, F., J. Bremen, M. C. Woussen-Colle, and J. De Graef. 1976. Failure of pentagastrin administration to restore postprandial acid secretion from Heidenhain pouches after antrectomy in dogs. Surgery (St. Louis). 80:586-590.

11. Walsh, J. H. 1974. Radioimmunoassay of gastrin. In Nuclear Medicine In Vitro. B. Rothfield, editor. J. B. Lippincott, Philadelphia. 231-248.

12. Johnson, L. R. 1981. Regulation of gastrointestinal growth. L. R. Johnson, editor. In Physiology of the Gastrointestinal Tract. Raven Press, New York, 169-196.

13. Card, W. I., and I. N. Marks. 1960. The relationship between the acid output of the stomach following "maximal" histamine stimulation and the parietal cell mass. Clin. Sci. (Lond.). 19:147-163.

14. Cox, A. J. 1952. Stomach size and its relation to chronic peptic ulcer. A.M.A. Arch. Pathol. 54:407-422.

15. Neuberger, P., M. Lewin, C. Recherche, S. Bonfils. 1972. Parietal and chief cell populations in four cases of the Zollinger-Ellison syndrome. Gastroenterology. 63:937-942.

16. Pearl, J. M., W. P. Ritchie, R. B. Gilsdorf, J. P. Delaney, and A. S. Leonard. 1966. Hypothalamic stimulation and feline gastric mucosal cellular populations. J. Am. Med. Assoc. 195:281-284.

17. Peters, M. N., and C. T. Richardson. 1983. Stressful life events, acid hypersecretion, and ulcer disease. Gastroenterology. 84:114-119.

18. Payne, R. A., and A. W. Kay. 1962. The effect of vagotomy on the maximal acid secretory response to histamine in man. Clin. Sci. (Lond.). 22:373-382.

19. Norgaard, R. P., D. E. Polter, J. W. Wheeler, and J. S. Fordtran. 1970. Effect of long term anticholinergic therapy in gastric acid secretion, with observations on the serial measurement of peak histalog response. Gastroenterology. 58:750-755.

20. Roland, M., A. Berstad, and I. Liavag. 1974. Acid and pepsin secretion in duodenal ulcer patients in response to graded doses of pentagastrin or pentagastrin and carbacholine before and after proximal gastric vagotomy. Scand. J. Gastroenterol. 9:511-518.

21. Feldman, M., and J. H. Walsh. 1982. Effect of bethanechol on gastric acid secretion and serum gastrin concentration after proximal gastric vagotomy. Ann. Surg. 196:14-17.

22. Hall, W. H., and G. P. Smith. 1969. Gastric secretory response to chronic hypothalamic stimulation in monkeys. Gastroenterology. $57: 491-499$. 